\title{
$\mathrm{KO}$
}

\section{KNOWLEDGE ORGANIZATION}

Official Bi-Monthly Journal of the International Society for Knowledge Organization

ISSN $0943-7444$

International Journal devoted to Concept Theory, Classification, Indexing and Knowledge Representation

\section{Contents}

\section{Articles}

Ole Olesen-Bagneux.

The Memory Library: How the Library

in Hellenistic Alexandria Worked.

Xiaoyue Ma and Jean-Pierre Cahier.

An Exploratory Study on Semantic Arrangement

of VDL-Based Iconic Knowledge Tags

Heather Lea Moulaison, Felicity Dykas,

and John M. Budd.

Foucault, the Author, and Intellectual Debt:

Capturing the Author-Function Through Attributes,

Relationships, and Events in Knowledge

Organization Systems .. .30

\section{Papers from the ISKO-UK Biennial Conference,}

"Knowledge Organization: Pushing the Boundaries,"

United Kingdom, 8-9 July, 2013, London

Fausto Giunchiglia, Biswanath Dutta,

and Vincenzo Maltese.

From Knowledge Organization to Knowledge

Representation

Elena Konkova, Ayşe Göker, Richard Butterworth, and Andrew MacFarlane.

Social Tagging: Exploring the Image, the Tags, and the Game
Andreas Oskar Kempf, Dominique Ritze, Kai Eckert, and Benjamin Zapilko.

New Ways of Mapping Knowledge Organization

Systems: Using a Semi-Automatic Matching Procedure

for Building up Vocabulary Crosswalks. .66

Tanja Svarre and Marianne Lykke.

Experiences with Automated Categorization

in E-Government Information Retrieval.. .76

\section{Brief Communication}

Ingetraut Dahlberg.

What is Knowledge Organization?

Emilia Currás.

The Nature of Information and Its Influence

in Human Cultures.

Books recently published.

Index to Volume 40 .97 


\title{
KNOWLEDGE ORGANIZATION
}

\author{
Official Bi-Monthly Journal of the International Society for Knowledge Organization \\ ISSN $0943-7444$ \\ International Journal devoted to Concept Theory, Classification, Indexing and Knowledge Representation
}

\section{Contents pages}

Olesen-Bagneux, Ole. The Memory Library: How the Library in Hellenistic Alexandria Worked. Knowledge Organization. 41(1), 3-13. 37 references.

\begin{abstract}
For millennia the famous library in Hellenistic Alexandria has been praised as an epicenter of enlightenment and wisdom. And yet, a question still seems unanswered: how was its literature classified and retrieved? It is a subject that has been given surprisingly little attention by the field of library-andinformation science-indeed, by scholarship in general. Furthermore, a certain way of thinking has influenced the few answers that have so far been attempted. It is as if the scholars of our era have tried to identify the modern, physical library in the Hellenistic library in Alexandria. But such an approach is biased in a basic way: It simply does not consider the impact of the cultural and intellectual context of the library. This article differs fundamentally, because I reject the notion that the library was like those of today. Accordingly, an entirely new way of understanding how the library actually worked, in terms of classification and retrieval processes is presented. The key element is to understand the library both as a physical structure and as a structure in the memory of the Alexandrian scholars. In this article, these structures are put together so as to propose a new interpretation of the library.
\end{abstract}

Ma, Xiaoyue and Cahier, Jean-Pierre. An Exploratory Study on Semantic Arrangement of VDL-Based Iconic Knowledge Tags. Knowledge Organization. 41(1), 14-29. 49 references.

Abstract: VDL (Visual Distinctive Language)-based iconic knowledge tags are graphically structured icons for knowledge representation. VDL was developed and assessed to enhance the connection of iconic tags and the connection of tagged knowledge. The purpose of this paper is to present further investigation on an arrangement method for these special tags as well as the characteristics of better tag presentation in knowledge organization systems (KOS). An online experiment was conducted to compare tagging results of four types of iconic tag presentations: two types of iconic tags (VDL-based iconic tags and iconic tags without explicit structure) under two arrangement methods respectively (random arrangement and semantic arrangement). Tagging quality and tagging speed were measured to identify how users locate and locate again appropriate iconic tags for knowledge tagging. A supplementary test on tag structure identification was also carried out for each tag presentation. Semantic arrangement of VDL-based icons helped users to tag given articles with more appropriate tags in less time. Users identified better tag structure in this type of tag presentation. This in-depth work of VDL-based iconic tags is among the first to investigate how to visually structure knowledge tags, a problem neglected by previous studies on icon knowledge representation.

Moulaison, Heather Lea, Dykas, Felicity, and Budd, John M. Foucault, the Author, and Intellectual Debt: Capturing the Author-Function Through Attributes, Relationships, and Events in Knowledge Organization Systems. Knowledge Organization. 41(1), 30-43. 24 references.

Abstract: Based on Foucault's exploration of the authorfunction, the current study investigates knowledge organization systems' (KOS's) treatment of persons who are also authors and the ability to record attributes, relationships and events related to those persons. FRBR and FRAD do well to extend the information in library authority records beyond the personal name as a character string to include attributes of the person, yet aspects of the person as an author and author-function can be enhanced. This paper begins with a discussion of the authorfunction as identified by Foucault and the complexities of identity that arise. Next, it reviews the Library and Information Science (LIS) literature on authorship and name authorities, then briefly discusses the current library content standard (Resource Description and Access, (RDA)) and the current library encoding standard, (MAchine Readable Cataloging, (MARC)). It then examines four projects making use of person data to enhance the author-function: Europeana, AustLit, The American Civil War: Letters and Diaries, and DBpedia. We conclude that additional attributes, relationships, and events are pivotal to moving toward more Foucault-friendly KOS's in libraries. Concerns with this more robust model of recoding information include the ethics of recording attributes of persons and problems of enduser searching in current systems.

Giunchiglia, Fausto, Dutta, Biswanath, and Maltese, Vincenzo. From Knowledge Organization to Knowledge Representation. Knowledge Organization. 41(1), 44-56. 34 references.

Abstract: So far, within the library and information science (LIS) community, knowledge organization (KO) has developed its own very successful solutions to document search, allowing for the classification, indexing and search of millions of books. However, current $\mathrm{KO}$ solutions are limited in expressivity as they only support queries by document properties, e.g., by title, author and subject. In parallel, within the artificial intelligence and semantic web communities, knowledge representation (KR) has developed very powerful end expressive techniques, which via the use of ontologies support queries by any entity property (e.g., the properties of the entities described in a document). 
However, KR has not scaled yet to the level of KO, mainly because of the lack of a precise and scalable entity specification methodology. In this paper we present DERA, a new methodology inspired by the faceted approach, as introduced in KO, that retains all the advantages of KR and compensates for the limitations of KO. DERA guarantees at the same time quality, extensibility, scalability and effectiveness in search.

Konkova, Elena , Göker, Ayşe, Butterworth, Richard, and MacFarlane, Andrew. Social Tagging: Exploring the Image, the Tags, and the Game. Knowledge Organization. 41(1), 57-65. 21 references.

\begin{abstract}
Large image collections on the Web need to be organized for effective retrieval. Metadata has a key role in image retrieval but rely on professionally assigned tags which is not a viable option. Current content-based image retrieval systems have not demonstrated sufficient utility on large-scale image sources on the web, and are usually used as a supplement to existing text-based image retrieval systems. We present two social tagging alternatives in the form of photo-sharing networks and image labeling games. Here we analyze these applications to evaluate their usefulness from the semantic point of view, investigating the management of social tagging for indexing. The findings of the study have shown that social tagging can generate a sizeable number of tags that can be classified as interpretive for an image, and that tagging behaviour has a manageable and adjustable nature depending on tagging guidelines.
\end{abstract}

Kempf, Andreas Oskar, Ritze, Dominique, Eckert, Kai, and Zapilko, Benjamin. New Ways of Mapping Knowledge Organization Systems: Using a Semi-Automatic Matching Procedure for Building up Vocabulary Crosswalks. Knowledge Organization. 41(1), 66-75. 25 references.

Abstract: Crosswalks between different vocabularies are an indispensable prerequisite for integrated, high-quality search scenarios in distributed data environments where more than one controlled vocabulary is in use. Offered through the web and linked with each other they act as a central link so that users can move back and forth between different online data sources. In the past, crosswalks between different thesauri have usually been developed manually. In the long run the intellectual updating of such crosswalks is expensive. An obvious solution would be to apply automatic matching procedures, such as the so-called ontology matching tools. On the basis of computer-generated correspondences between the Thesaurus for the Social Sciences (TSS) and the Thesaurus for Economics (STW), our contribution explores the trade-off between IT-assisted tools and procedures on the one hand and external quality evaluation by domain experts on the other hand. This paper presents techniques for semiautomatic development and maintenance of vocabulary crosswalks. The performance of multiple matching tools was first evaluated against a reference set of correct mappings, then the tools were used to generate new mappings. It was concluded that the ontology matching tools can be used effectively to speed up the work of domain experts. By optimizing the workflow, the method promises to facilitate sustained updating of high-quality vocabulary crosswalks.

Svarre, Tanja and Lykke, Marianne. Experiences with Automated Categorization in E-Government Information Retrieval. Knowledge Organization. 41(1), 76-84. 35 references.

Abstract: High-precision search results are essential for supporting e-government employees' information tasks. Prior studies have shown that existing features of e-government retrieval systems need improvement in terms of search facilities (e.g., Goh et al. 2008), navigation (e.g., de Jong and Lentz 2006) and metadata (e.g., Kopackova, Michalek and Cejna 2010). This paper investigates how automated categorization can enhance information organization and retrieval, and presents the results of a realistic evaluation that compared automated categorization with free text indexing of the government intranet used by Danish tax authorities. The evaluation demonstrates a potential for automated categorization in a government context. In terms of quantitative measures free text indexing performed at the same level or better than searching by categorization. However, the qualitative analysis revealed that categorized overviews were useful if the participant did not possess much knowledge of the task at hand. When task knowledge was present, categorization was used to support the assumptions of a correct search. Participants avoided automated categorization if high-precision documents were among the top results or if few documents were retrieved. The findings emphasise the importance of simultaneous search options for e-government IR systems, and reveal that automated categorization is valuable in improving search facilities in e-government.

Dahlberg, Ingetraut. Brief Communication: What is Knowledge Organization? Knowledge Organization. 41(1), 85-91. 27 references.

Abstract: As an introduction, the circumstances leading to the foundation of the International Society for Knowledge Organization (ISKO) are outlined and the prerequisites for the formal and conceptual description of the scope of knowledge organization (KO) are laid out, followed by the explanation of the scheme as used in the bibliography of KO. An overview is provided of the tasks and activities of this discipline; thereafter and in conclusion an urgent appeal is made to ISKO and to all active scientific societies with a view to establishing $\mathrm{KO}$ as an autonomous scientific discipline within the science of science, as well as an indication is given of urgently required tasks. 


\section{KNOWLEDGE ORGANIZATION}

International Journal devoted to Concept Theory, Classification, Indexing and Knowledge Representation

Currás, Emilia. Brief Communication: The Nature of Information and Its Influence in Human Cultures. Knowledge Organization. 41(1), 92-96. 17 references.

Abstract: Starting this paper by discussing the nature of information, where it comes from and its uses, highlighting the importance of its characteristic to be the prime element in human development. It also refers to the ways in which we understand information, whether as a process or as a phenomenon and, therefore, emphasises its attributes as a means of accessing science, wisdom and the truth. Information as such does not have an identity of its own, it has to be upheld ... by an object, be it material or electronic. Here also it refers to its features, such as instability, inconsistency, perpetuity, etc. A new theory of knowledge is formulating, where information is taking as its paradigm, which is named "Informationisms" and it is an original idea of the author. The influence of information is studied as a prime element in human culture. Information is the first element in the development of the individual and, indeed, as well, in the development of humankind as a whole. It is giving us a general overview of the future influence of the different types of cultures, both humanistic and technological ones. The importance of information is so that it could become a factor in the human race annihilation from the Earth planet, but human beings have decided to transform their way of life and the direction in which their civilization should be conducted. 